\title{
Pós-Graduação em Psicologia na PUC-Campinas: dissertações e teses (1975-2004)
}

\author{
Post Graduation in Psychology at PUC-Campinas: \\ theses and dissertations (1975-2004)
}

\author{
Geraldina Porto WITTER ${ }^{1,2}$
}

\section{Resumo}

A avaliação da produção científica é uma atividade que apresenta muitas utilidades em cientometria e em política educacional e científica. O artigo apresenta uma avaliação quantitativa da produção de dissertações e teses defendidas na Pontifícia Universidade Católica de Campinas, de 1975 até 2004, isto é, por 30 anos. Os resultados mostraram 577 trabalhos no nível de mestrado e 93 no de doutorado, totalizando 670 produtos científicos. A primeira dissertação de mestrado foi defendida em 1975 e a primeira tese em 1997. Predomina a autoria feminina (81,79\%). Alguns orientadores tiveram uma maior participação total possivelmente em decorrência do número de anos vinculados ao programa e do regime de trabalho assumido na Universidade.

Palavras-chave: cientometria; produção científica; psicologia clínica; psicologia escolar.

\begin{abstract}
The evaluation of scientific production is an important activity to the scientific and educational politics and patterns. This paper presents a quantitative evaluation about the theses and dissertations that had been presented at the Pontificia Universidade Católica de Campinas, from 1975 to 2004, that is, 30 years. The results shows 577 papers from master degree and 93 dissertations (PhD), which amounts to 670 scientific productions. The first master thesis was defended in 1975, and only in 1997 the PhD first thesis was defended. The feminine authorship has been the most common (81,79\%). Some advisers' total participation have been more intense than the others, according to these professionals' career as a member of this program.
\end{abstract}

Key words: scientometry; scientific research; clinical psychology; school psychology.

Este texto apresenta uma breve análise da produção de dissertações e teses defendidas na pós-graduação da Pontifícia Universidade Católica de Campinas (PUC-Campinas) ao longo de 30 anos de intenso trabalho e dedicação de professores-orientadores, alunos e funcionários. Para contextualizar historicamente essa produção, uma breve perspectiva do seu evoluir é inicialmente apresentada.

No Brasil, os cursos de mestrado e de doutorado são relativamente novos quando comparados ao mundo europeu, a vários países das Américas e do Oriente. Assim sendo, no Brasil, 30 anos de produção

\section{$\boldsymbol{\nabla \nabla \nabla \nabla}$}

1 Programa de Pós-Graduação em Psicologia, Centro de Ciências da Vida, Pontifícia Universidade Católica de Campinas. Av. John Boyd Dunlop, s/n, Prédio Administrativo, Jd. Ipaussurama, 13060-904, Campinas, SP, Brasil.

2 Universidade de Mogi das Cruzes. Mogi das Cruzes, SP, Brasil.E-mail: < pwitter@umc.br>.

Agradecimentos: a Ricardo de Freitas Domingos, que colaborou como auxiliar de pesquisa. 
em uma dada instituição é motivo para brindá-la pelo feito. Considerou-se relevante, como comemoração, fazer uma retrospectiva dessa trajetória.

\section{Perspectiva histórica}

No começo dos anos setenta, estimuladas pelo Governo, que se mostrou ciente da relevância da perspectiva para o desenvolvimento do país e da necessidade de contar com recursos humanos de alto nível, as universidades brasileiras começaram a investir na pós-graduação de forma sistemática.

A Pontifícia Universidade Católica de Campinas, ciente dessas necessidades, envidou esforços nesse sentido e um dos resultados foi a criação do Mestrado em Psicologia Clínica em 1972.

De acordo com Monte Serrat (1992), desde o início estavam presentes dois enfoques: o psicanalítico e o comportamental. O credenciamento e o recredenciamento do curso foram uma constante ao longo dos anos. Mais tarde, com a ampliação do corpo docente da pós-graduação e continuando uma forte tradição da graduação voltada para a área educacional, já firmada em cursos de especialização, surgiu o mestrado em Psicologia Escolar (1990), conforme relata Witter (1991). Mais tarde, ao se ter consolidado um trabalho nos cursos de mestrado, o programa de pós-graduação da PUC-Campinas, na área de Psicologia, criou, em 1995, o seu doutorado em ciências, concentrando-se na área: Psicologia como profissão e ciência (Domingos, 1999).

A pós-graduação em Psicologia na PUC-Campinas acompanhou o evoluir da pós-graduação no Brasil, que vem crescendo lentamente face às necessidades requeridas para um desenvolvimento efetivo do país. Embora os dados brutos possam parecer satisfatórios, há muitas áreas do conhecimento em geral e da Psicologia em particular que estão em descoberto.

De acordo com a Coordenação de Aperfeiçoamento de Pessoal de Nível Superior (Capes) (2002, www.capes.gov.br), no começo do atual século o Brasil contava com 1570 cursos, incluindo mestrados acadêmicos, mestrados profissionalizantes e doutorados. A distribuição dos cursos estava longe de contemplar de forma similar as várias regiões geográficas. Alguns dados podem servir de referência. Dos 99338 estudantes,
67919 estavam na Região Sudeste, 16303 na Sul, 4301 na Centro-Oeste e 1350 na Região Nordeste.

Titularam-se em 2002: 22735 mestres, 686 mestres profissionais e 6843 doutores. Esses números sugerem insuficiência até mesmo para preencher as necessidades de composição dos quadros docentes dos cursos de graduação, caso, como é desejável, fosse realmente exigida titulação para assumir a docência no ensino superior, como ocorre nos países mais avançados (Witter, 2004).

Os programas de pós-graduação estavam concentrados nas escolas oficiais (8690), sendo 56\% em instituições federais, 30\% em estaduais e 2\% em municipais. As instituições privadas respondiam por 14\% dos cursos de pós-graduação no Brasil em 2002; dentre elas, cinco eram confessionais, como é a PUC-Campinas. As confessionais eram responsáveis por 40\% das vagas disponíveis no setor privado (Instituto Nacional de Estudos e Perspectivas Educacionais, 2004).

\section{Dissertações e teses de Psicologia defendi- das na PUC-Campinas}

A análise de produção de um programa pode fornecer informações sobre sua evolução histórica e as tendências. Ao mesmo tempo que se visualiza a matéria produzida, são disponibilizados dados úteis à administração e ao planejamento, tomada de decisão quanto à implantação e à extinção de linha de pesquisa (Witter, 2005).

A produção de dissertações e de teses da PUC-Campinas já foi objeto de vários estudos de metaciência, os quais enfocaram todos os produtos ou temas específicos, centraram-se exclusivamente na sua produção ou a compararam com a de outras universidades. Aqui são apontadas algumas dessas pesquisas. Possivelmente, a primeira delas foi o trabalho de Witter (1986), que analisou a produção da Universidade de São Paulo (USP), PUC-Campinas e Pontifícia Universidade Católica de São Paulo (PUC-São Paulo) na década de setenta do século passado (1970-1979). Muitos outros seguiram essa mesma linha, como, por exemplo, Domingos (1999), que analisou a produção de cursos de pós-graduação em Psicologia incluindo a PUC-Campinas. 
Um estudo de avaliação exclusiva de aspecto da produção - stress da PUC-Campinas - é o de Cusatis Neto e Andrade (2005). O trabalho apresenta uma análise da produção de dissertações e teses defendidas na pós-graduação de Psicologia da instituição, de 1984 até junho de 2004, totalizando 44 produtos. Verificaram-se predomínio de autoria feminina (94,5\% dos mestrados e 100\% dos doutorados); predomínio de delineamento descritivo nas dissertações (61\%) e de experimentação (100\%) nas teses; os adultos foram o principal alvo de estudo, sendo de se destacar também o stress associado a doenças.

Aqui será apresentada uma breve análise quantitativa das dissertações e teses defendidas na PUC-Campinas desde a sua primeira ocorrência (1975) até completar os 30 anos de produção (2004).

Em dois de dezembro de 1975, com a orientação de La Puente, ocorreu na PUC-Campinas, no mestrado em Psicologia Clínica, a primeira defesa de dissertação. O trabalho intitulado "Uma experiência de grupo de encontro básico com jovens sujeitos farmacodependentes" é de autoria de Sônia M.R. Wolf. Desde então a produção cresceu e as defesas se sucederam com regularidade.

Mesmo antes de funcionar o mestrado em Psicologia Escolar, na área clínica, algumas dissertações se voltaram para questões educacionais e eram indícios da necessidade de abrir espaço para a formação na área escolar. A primeira defesa desse tipo de trabalho foi o orientado por Néri e defendido em 1978 por llka V. Moroni, que analisava o efeito de duas variáveis internas em um curso programado aplicado em escola secundária (hoje, ensino médio).

A primeira defesa oficial de dissertação do mestrado de Psicologia Escolar foi a relativa ao trabalho de Francisco A.F. Oliveira, sobre a formação do psicólogo escolar, em 3 de julho de 1992, sob a orientação de Witter.

No nível de doutorado, a primeira tese a ser defendida na PUC-Campinas também ocorreu na Psicologia, em 23 de junho de 1997, dia em que Otávio M.L. Mendonça defendeu seu trabalho que compreendeu, na primeira parte, uma análise de produção sobre o ensino de ciências e, na segunda, o teste de eficiência de um programa de ensino de ciências, na escola fundamental rural, no interior do estado da Paraíba (Cariri), que se mostrou econômico e de alta eficiência.

A Tabela 1 apresenta a ocorrência das dissertações e teses defendidas na PUC-Campinas ao longo dos 30 anos aqui enfocados. Registrou-se uma produção crescente com alguma flutuação, possivelmente em decorrência do término de vigência de bolsas e mudanças no quadro de orientadores.

Considerando-se que o primeiro mestrado foi defendido no final de 1975, a média nos 30 anos foi de 19 dissertações por ano. Entretanto, o processo acelerou-se com o início da produção na área da Psicologia Escolar. A produção do doutorado começou em 1997,

Tabela 1. Dissertações e teses defendidas na PUC-Campinas (1975-2004).

\begin{tabular}{|c|c|c|c|}
\hline Ano & Mestrado & Doutorado & Total \\
\hline 1975 & 1 & & 1 \\
\hline 1976 & 3 & & 3 \\
\hline 1977 & 4 & & 4 \\
\hline 1978 & 7 & & 7 \\
\hline 1979 & 6 & & 6 \\
\hline 1980 & 9 & & 9 \\
\hline 1981 & 8 & & 8 \\
\hline 1982 & 15 & & 15 \\
\hline 1983 & 10 & & 10 \\
\hline 1984 & 8 & & 8 \\
\hline 1985 & 8 & & 8 \\
\hline 1986 & 11 & & 11 \\
\hline 1987 & 8 & & 8 \\
\hline 1988 & 10 & & 10 \\
\hline 1989 & 4 & & 4 \\
\hline 1990 & 18 & & 18 \\
\hline 1991 & 15 & & 15 \\
\hline $1992\left({ }^{*}\right)$ & 17 & & 17 \\
\hline 1993 & 30 & & 30 \\
\hline 1994 & 33 & & 33 \\
\hline 1995 & 34 & & 34 \\
\hline 1996 & 31 & & 31 \\
\hline $1997\left(^{* *}\right)$ & 50 & 1 & 51 \\
\hline 1998 & 30 & 1 & 31 \\
\hline 1999 & 30 & 14 & 44 \\
\hline 2000 & 25 & 14 & 39 \\
\hline 2001 & 35 & 12 & 47 \\
\hline 2002 & 32 & 14 & 46 \\
\hline 2003 & 44 & 24 & 68 \\
\hline 2004 & 41 & 13 & 54 \\
\hline Total & 577 & 93 & 670 \\
\hline
\end{tabular}

(*) Início da produção no mestrado de Psicologia Escolar; $\left(^{* *}\right)$ Início da produção no doutorado. 
não tendo ainda completado uma década. No período aqui considerado foi de 1,6 a produção média de teses. Ao longo dos 30 anos a média de produção foi de 22 documentos. Considerando-se as dimensões do corpo docente e discente, pode-se dizer que se está diante de um bom volume de produção. Além disso, estudos anteriores como os de Domingos (1999) e Lima (1999) atestam a qualidade dessa produção em estudos comparativos.

Muitos orientadores contribuíram para essa produção. Alguns permaneceram por mais tempo no quadro de docentes, outros colaboraram como orientadores externos, mas todos concorreram para que a produção se efetivasse. O registro do número de orientações efetivadas por orientador aparece na Tabela 2. Ao todo foram 45 os orientadores responsáveis pelas dissertações e teses.

Destacaram-se alguns orientadores de maior número de trabalhos de mestrado defendidos: Lipp $(n=61)$, Terzis $(n=54)$, Witter $(n=52)$ e Amaral $(n=49)$. Em relação às teses, as maiores produções foram da responsabilidade de Witter ( $n=18)$, Guzzo ( $n=12)$ e com igual número $(n=9)$ Wechesler e Lipp. No total, os produtores com maior número de produtos foram Lipp e Witter com 70 orientandos cada uma, vindo a seguir Terzis ( $n=62)$, Guzzo ( $n=53)$ e Amaral ( $n=51)$. Provavelmente, o regime de trabalho e o número de anos atuando no programa são variáveis que influem nesse resultado e que merecem outros estudos (Tabela 2).

Destacou-se para análise o gênero dos autores das dissertações e teses (Tabela 3). Os dados obtidos confirmaram a tendência da amostra de Cusatis Neto e Andrade (2005) de predomínio das mulheres, refletindo o que ocorre na própria formação e na área no Brasil: a profissão aparece como predominantemente feminina. Vale lembrar que já na década de setenta Witter (1986) observou tal tendência.

No nível de mestrado, 18,02\% dos autores eram do gênero masculino e $81,97 \%$ do feminino, tendo a diferença valor estatístico já que $\chi^{2}=235,98$ para $\chi_{c}^{2}=3,84$ (n.g.l. $=1$ e n.sig=0,05). No doutorado também ocorreu significância, pois $\chi_{0}^{2}=34,93$. No total o valor obtido foi de 296,88 . Esses resultados também estão consoantes com os de Witter (1991), Nakano (2005) sobre dissertações e teses arroladas na CAPES sobre criatividade e de Witter e Assis Maria (2005) sobre idosos na mesma base bibliográfica.

Tabela 2. Participação de docentes na orientação de dissertação e teses na PUC-Campinas (1975-2004).

\begin{tabular}{|c|c|c|c|}
\hline Orientador & Mestrando & Doutorando & Total \\
\hline Aardweg, J.M. van den & 3 & & 3 \\
\hline Amaral, V.L.A.R & 49 & 2 & 51 \\
\hline Amatuzzi, M.M. & 25 & 6 & 31 \\
\hline Andrade, A.M.O. & 5 & & 5 \\
\hline Betioli, J.O. & 1 & & 1 \\
\hline Bittencourt, W.B.F. & 2 & & 2 \\
\hline Boren, J.J. & 2 & & 2 \\
\hline Botomé, S.P. & 1 & & 1 \\
\hline Campos, L.F.L. & 7 & & 7 \\
\hline Carvalho, R.M.L.L. & 22 & 8 & 30 \\
\hline Cassorla, R.M.S. & 2 & & 2 \\
\hline Cury, V.E. & 18 & 2 & 20 \\
\hline Duran, A.P. & 1 & & 1 \\
\hline Ferreira, M.E.R. & 1 & & 1 \\
\hline Fiamenghi, Jr. G. & 5 & & 5 \\
\hline Fish, J.M. & 3 & & 3 \\
\hline Góes, M.C.R. & 1 & & 1 \\
\hline Guzzo, R.L.S. & 41 & 12 & 53 \\
\hline Jaequimim, A. & 1 & & 1 \\
\hline Kerbauy, R.R. & 1 & & 1 \\
\hline Knobel, M. & 21 & & 21 \\
\hline Kpfuffer, M.C.M. & 1 & & 1 \\
\hline La Puente, M. & 8 & & 8 \\
\hline Lakatos, E.M. & 1 & & 1 \\
\hline Laloni, D.T. & 1 & & 1 \\
\hline Leite, T.M.M. & 1 & & 1 \\
\hline Lipp. M.E.N. & 61 & 9 & 70 \\
\hline Martins Filho, J. & 1 & & 1 \\
\hline Neri, A.L. & 25 & & 25 \\
\hline Nogueira, J.C. & 1 & & 1 \\
\hline Oliveira, N.H.M.A. & 12 & 1 & 13 \\
\hline Pfromm Netto, S. & 32 & 6 & 38 \\
\hline Rosa, J.T. & 2 & & 2 \\
\hline Rosado, E. & 20 & 5 & 25 \\
\hline Sadalla, A. M. F. A. & 4 & & 4 \\
\hline Silva, M.E.L. & 14 & 1 & 15 \\
\hline Simões, J.C. & 1 & & 1 \\
\hline Tápia, LE.R. & 1 & & 1 \\
\hline Terzis, A. & 54 & 8 & 62 \\
\hline Tonelotto, J.M.F. & 9 & & 9 \\
\hline Trinca, W. & 8 & & 8 \\
\hline Wechsler, S.M. & 33 & 9 & 42 \\
\hline Witter, G.P. & 52 & 18 & 70 \\
\hline Wood, J.K. & 2 & & 2 \\
\hline Yoshida, E.M.P. & 21 & 6 & 27 \\
\hline Total & 577 & 93 & 670 \\
\hline
\end{tabular}


Outra variável estudada foi o título das dissertações e das teses (Tabelas 4 e 5), enfocando-se o número de vocábulos, conforme apresentado a seguir.

O título do trabalho científico é o primeiro contato do leitor com o texto, pode atraí-lo ou afastá-lo de acordo com a motivação e com a necessidade de informação. O título deve ser claro, correto e informar o leitor com precisão sobre o que trata o texto, já que os vocábulos substanciais do texto são usados para inserir o trabalho nas bases bibliográficas. Títulos fantasias podem até ser comercialmente relevantes para a promoção de venda de livros, mas não devem ser usados em trabalhos científicos, dos quais devem ser esperadas informações precisas sobre o assunto e variáveis envolvidas.

Uma das características dos títulos é o número de vocábulos. Espera-se que o autor seja suficientemente capaz e criativo para, usando doze ou mesmo dez vocábulos, transmitir ao seu leitor uma respectiva geral do que trata o trabalho. Alguns eventos e mesmo periódicos aceitam no máximo doze palavras, que, segundo as pesquisas, atendem as necessidades nas várias línguas.

Tabela 3. Gênero dos autores de dissertações e teses da PUC-Campinas.

\begin{tabular}{|c|c|c|c|c|c|c|}
\hline \multirow{2}{*}{ Gênero } & \multicolumn{2}{|c|}{ Mestrado } & \multicolumn{2}{|c|}{ Doutorado } & \multicolumn{2}{|c|}{ Total } \\
\hline & $n$ & $\%$ & $n$ & $\%$ & $n$ & $\%$ \\
\hline Masculino & 104 & 18,03 & 18 & 19,35 & 122 & 18,21 \\
\hline Feminino & 473 & 81,97 & 73 & 80,65 & 548 & 81,79 \\
\hline Total & 577 & 100,00 & 93 & 100,00 & 670 & 100,00 \\
\hline
\end{tabular}

Tabela 4. Número de vocábulos nos títulos das teses da PUC-Campinas.

\begin{tabular}{|c|c|c|c|c|c|}
\hline \multirow{2}{*}{ Anos } & \multicolumn{4}{|c|}{ Vocábulos } & \multirow{2}{*}{ Tota } \\
\hline & 0 a 5 & 6 a 12 & 13 a17 & $>17$ & \\
\hline 1997 & & 1 & & & 1 \\
\hline 1998 & & 1 & & & 1 \\
\hline 1999 & 1 & 12 & 1 & & 14 \\
\hline 2000 & 1 & 12 & 1 & & 14 \\
\hline 2001 & 2 & 9 & 1 & & 12 \\
\hline 2002 & & 10 & 3 & 1 & 14 \\
\hline 2003 & & 21 & 3 & & 24 \\
\hline 2004 & & 8 & 3 & 2 & 13 \\
\hline Total & 4 & 74 & 12 & 3 & 93 \\
\hline
\end{tabular}

Foi feito um estudo dos títulos das dissertações e teses aqui enfocadas quanto ao número de vocábulos usados pelos autores (Tabela 4 e 5). No que concerne aos doutorados, os dados apresentados na Tabela 4 evidenciam que em todos os anos os títulos estiveram predominantemente na classe esperada de seis a doze vocábulos, sendo o $n=74$, vindo a seguir com $n=12$ os que se encaixam na classe de até cinco. Esses resultados indicam que se está seguindo padrões internacionais e esperados (74\%). Estima-se que cada vez mais esse aspecto seja devidamente cuidado, sendo particularmente importante a atenção a títulos demasiado longos, pois muitos não podem ser devidamente registrados nas bases bibliográficas, além disso, com conhecimento e criatividade sempre é possível reduzi-los ao padrão esperado.

Tabela 5. Número de vocábulos nos títulos das dissertações da PUC-Campinas.

\begin{tabular}{|c|c|c|c|c|c|}
\hline \multirow{2}{*}{ Anos } & \multicolumn{4}{|c|}{ Vocábulos } & \multirow{2}{*}{ Total } \\
\hline & 0 a 5 & 6 a 12 & 13 a17 & $>17$ & \\
\hline 1975 & & 1 & & & 1 \\
\hline 1976 & & 3 & & & 3 \\
\hline 1977 & & 3 & & 1 & 4 \\
\hline 1978 & & 5 & 1 & 2 & 8 \\
\hline 1979 & 2 & 3 & 1 & 2 & 8 \\
\hline 1980 & & 4 & 2 & 3 & 9 \\
\hline 1981 & & 4 & 2 & 2 & 8 \\
\hline 1982 & & 7 & 5 & 3 & 15 \\
\hline 1983 & 2 & 1 & 5 & 2 & 10 \\
\hline 1984 & 1 & 3 & 2 & 2 & 8 \\
\hline 1985 & & 3 & 4 & 1 & 8 \\
\hline 1986 & 1 & 3 & 5 & 3 & 12 \\
\hline 1987 & & 3 & 4 & 1 & 8 \\
\hline 1988 & & 4 & 4 & 2 & 10 \\
\hline 1989 & & 2 & 2 & & 4 \\
\hline 1990 & & 7 & 8 & 3 & 18 \\
\hline 1991 & & 9 & 3 & 3 & 15 \\
\hline 1992 & & 11 & 4 & 2 & 17 \\
\hline 1993 & & 13 & 13 & 4 & 30 \\
\hline 1994 & & 23 & 7 & 3 & 33 \\
\hline 1995 & & 26 & 7 & 1 & 34 \\
\hline 1996 & 1 & 29 & 1 & & 31 \\
\hline 1997 & 2 & 45 & 3 & & 50 \\
\hline 1998 & & 20 & 5 & & 25 \\
\hline 1999 & & 28 & 2 & & 30 \\
\hline 2000 & 2 & 20 & 3 & & 25 \\
\hline 2001 & 1 & 30 & 5 & & 36 \\
\hline 2002 & 1 & 27 & 4 & & 32 \\
\hline 2003 & 5 & 35 & 4 & & 44 \\
\hline 2004 & & 33 & 8 & & 41 \\
\hline Total & 18 & 405 & 114 & 40 & 577 \\
\hline
\end{tabular}


No que diz respeito aos trabalhos de mestrado (Tabela 5), a maior ocorrência foi dos trabalhos com títulos entre seis e doze vocábulos, que alcançaram a freqüência de 405 dissertações, ou seja, 70,19\%. Portanto, a maioria respeitou o padrão desejado. Observou-se que desde 1997 desapareceram os títulos demasiado longos, mas há ainda espaço para aperfeiçoamento. Também caberia um estudo sobre a qualidade dos títulos, implicando a análise da relação objetivométodo, da precisão, do índice de informação e da ausência de títulos-fantasia.

Foi feita uma análise estatística para verificar se havia diferenças quanto ao número de vocábulos (Ho: $\chi^{2}=0$, Ha: $\chi^{2} \neq 0$, n.sig. $=0,05$, n.g.l. $=3$ e $\chi_{c}^{2}=7,81$ ). Foi obtido $\chi^{2} \mathrm{o}=808,51$, ou seja, pode-se concluir que a concentração na categoria esperada $(579,30)$ foi significativa posto que Ho foi rejeitada.

No aspecto considerado, pode-se afirmar que os títulos das dissertações e teses estão dentro dos parâmetros cientificamente desejados.

\section{Conclusão}

Essa perspectiva histórica, 30 anos de caráter quantitativo, enfocando a produção de dissertações e teses na área de Psicologia da Pontifícia Universidade Católica de Campinas, permite concluir que:

- a PUC-Campinas vem contribuindo sistematicamente com dissertações e teses que atendem aos quesitos de boa produção;

- a autoria dos trabalhos é predominantemente feminina;

- a diversificação de área (clínica + escolar) e posteriormente a inclusão do nível de doutorado refletiram positivamente na produtividade do programa e

- alguns orientadores tenderam, por contingências diversas, a produzir mais do que outros.

Vale lembrar que alguns ficaram no programa por pouco tempo, seus nomes apareceram ligados apenas aos primeiros produtos, outros ficaram por muitos anos e lá permanecem. Novos orientadores estão preenchendo os espaços decorrentes do afastamento de vários docentes. Tudo indica que o programa está empenhado em evoluir.

\section{Referências}

Cusatis Neto, R., \& Andrade, V.G. (2005). Análise da produção de stress na Pontifica Universidade Católica de Campinas. In M.E.N. Lipp (Org). Ostress no Brasil:pesquisas avançadas (pp.197-204). Campinas: Papirus

Domingos, N.A.M. (1999). Produção científica: análise de resumos de dissertação e teses em Psicologia (1992/1996). Tese de doutorado não-publicada, Pontifícia Universidade Católica de Campinas.

Instituto Nacional de Estudos e Perspectiva Educacionais Anísio Teixeira (INEP). (2004). Censo de educação superior: 2003 [Resumo Técnico]. Brasília: INEP.

Lima, M.F. (1999). A psicologia em teses de administração escolar. Tese de doutorado em Psicologia não-publicada, Pontifícia Universidade Católica de Campinas.

Mendonça, O.M.L. (1997). Ensino-aprendizagem de ciência: produção e aplicação. Tese de doutorado não-publicada, Pontifícia Universidade Católica de Campinas.

Monte-Serrat, S. (1992). 20 anos de Pós-Graduação. Estudos de Psicologia, 9 (1), 141-147.

Moroni, I.V. (1978). Curso programado em escola secundária: análise de duas variáveis internas. Dissertação de mestrado em Psicologia Clínica não-publicada, Pontifícia Universidade Católica de Campinas.

Nakano, T.C. (2005). Pesquisa em criatividade: analise da produção científica do banco de teses da CAPES (1996-2001). In G.P. Witter. Metaciência e psicologia. Campinas: Alínea.

Oliveira, F.A.F. (1992). Formação do psicólogo escolar em duas instituições de ensino superior: análise através dos planos de disciplinas. Dissertação de mestrado em Psicologia não-publicada, Pontifícia Universidade Católica de Campinas.

Witter, G.P. (1986). Uma década (1970-1979) de Psicologia: adendos à história da Psicologia no Brasil. In J.L.O. Bueno. O estudo do comportamento: pesquisa e prática no Brasil. Ribeirão Preto: Sociedade de Psicologia de Ribeirão Preto.

Witter, G.P. (1991). A pós-graduação em Psicologia na PUCCAMP-1990. Estudos de Psicologia, 8, 157-159.

Witter, G.P. (2005). Metaciência e psicologia. Campinas: Alínea.

Witter, G.P., \& Assis Maria, F. (2005). Velhice no Banco de Teses da CAPES (2000 e 2001). In G.P. Witter. Metaciência e Psicologia. Campinas: Alínea.

Wolf, S.M.R. (1975). Uma experiência de grupo de encontro básico com jovens sujeitos farmacodependentes. Dissertação de mestrado em Psicologia Clínica não-publicada, Pontifícia Universidade Católica de Campinas.

Recebido para publicação em 3 de agosto e aceito em 5 de setembro de 2005. 